\title{
NOTES
}

\section{A microcomputer interface to control reinforcement delivery}

HENRY BLEKKENHORST

Great Lake Forest Products, Thunder Bay, Ontario, Canada

and

\section{STEPHEN R. GOLDSTEIN}

Lakehead University, Thunder Bay, Ontario, Canada

A method for interfacing a PET microcomputer to an operant arena is described.

An operant arena for the study of dispersion patterns was described by Goldstein (1981). Software programs by which a PET microprocessor controls and analyzes schedule performance and by which a VAX computer analyzes dispersion patterns were described by Goldstein, Blekkenhorst, and Mayes (1982). This note concerns the hardware used to interface the PET microprocessor to the operant arena.

Figure 1 shows a circuit to trigger a pellet dispenser and a circuit to monitor a set of bar contacts. The PET controls eight of these circuits. The arrangement as described could also be used to control separate operant chambers containing from one to eight manipulanda and dispensers.

Two parallel $\mathrm{I} / \mathrm{O}$ ports on the PET are used to detect responses and to control reinforcements. The interface to the arena consists of isolating buffers for the bar contacts and current drivers for the dispensers. The IEEE port is used as an output port (address \$E823) to deliver a pulse to the dispenser-triggering circuit. The second port (address $\$ \mathrm{E} 84 \mathrm{~F}$ ) is used as an input port to read the state of the bars. Each of the eight lines on this port is connected to the circuit that monitors a set of bar contacts. The circuit serves to suppress contact bounce and to isolate the input port.

The circuit used to trigger the dispenser receives a low pulse from a line on the PET IEEE port. This pulse

This work was submitted in partial fulfillment of the requirements for a Bachelor of Engineering degree by the senior author. This research was supported, in part, by the National Science and Engineering Research Council (NSERC) and the President's NSERC fund. Reprint requests should be sent to Stephen R. Goldstein, Department of Psychology, Lakehead University, Thunder Bay, Ontario P7B 5E1, Canada.

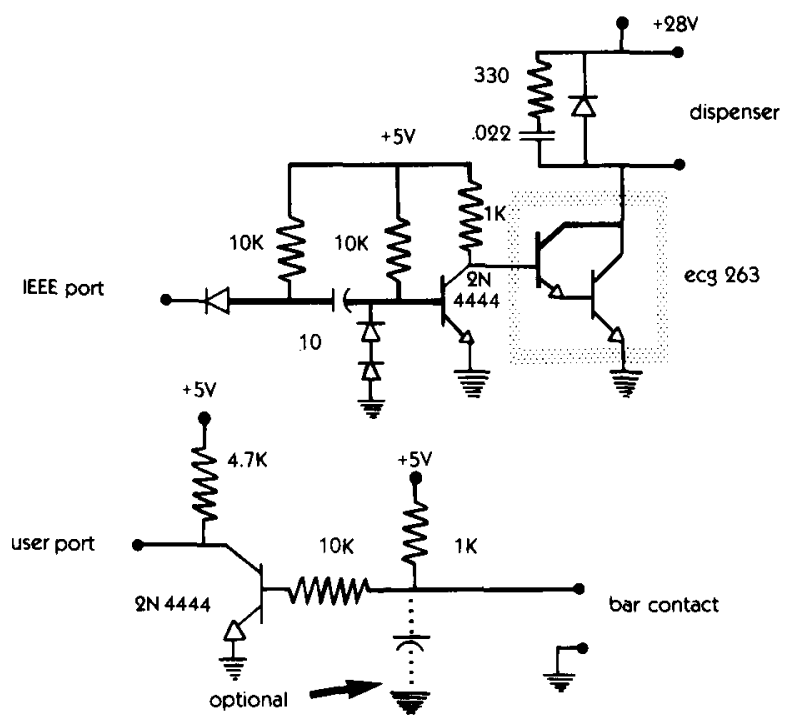

Figure 1. Circuit diagram for one of the eight interface units between the operant equipment and the PET. All resistors are rated in ohms; all capacitors are rated in microfarads.

switches on the current to the stepper motor in the food dispenser. Since the dispensers are rated at $25 \%$ duty cycle, the output circuit also limits the pulse to a length of $250 \mathrm{msec}$. A resistor-capacitor-diode network is included to suppress the spike created when the dispenser is activated.

The stepper motor in the dispenser requires a current of 2 A from a $28-\mathrm{V}$ supply. The driver circuit allows the TTL level signal to switch the 2-A drive current for the dispensers. Darlington power transistors are used as the switch. These have a current gain of at least 2,000 , so an input current of $2 \mathrm{~mA}$ can control the output current of $2 \mathrm{~A}$. This method requires that the signal ground be common to the $28 \cdot \mathrm{V}$ supply.

\section{REFERENCES}

Goldstein, S. R. An operant arena for rats. Behavior Research Methods \& Instrumentation, 1981, 13, 37-39.

Goldstein, S. R., Blekkenhorst, H. J., \& Mayes, L. S. Computerization of the operant arena. Behavior Research Methods \& Instrumentation, 1982, 14, 369-372.

(Manuscript accepted for publication March 22, 1983.) 